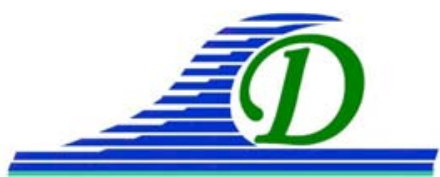

\title{
Evolution des plages de poche de Corse : vers un modèle conceptuel
}

\section{Rémi BELON $^{1}$, Yann BALOUIN ${ }^{2}$, Anne MEROUR ${ }^{1}$, Camille RIOTTE ${ }^{1}$}

1. BRGM, Direction Régionale Corse, Immeuble Agostini, ZI de Furiani, 20600 Bastia, France.r.belon@brgm.fr

2. BRGM, Direction Régionale Languedoc-Roussillon, 1039, rue de Pinville, 34000 Montpellier, France.y.balouin@brgm.fr

\section{Résumé :}

Dans cette étude, les résultats de l'analyse de l'évolution de 44 plages de poche de Haute-Corse sont présentés. Les plages de poche sont des plages de faible étendue, n’excédant généralement pas le kilomètre, abritées en fond de baie. Les comportements observés le plus fréquemment sont ceux entrainant une rotation de la direction prédominante de la plage en fonction de la direction de la houle incidente. L'absence de marée significative et la présence de faibles courants côtiers amènent à penser que les changements morphologiques de ces plages sont dus principalement aux évènements extrêmes. Cependant, la variabilité des évolutions constatées indique que d'autres facteurs jouent également un rôle dans ces évolutions côtières. Pour évaluer ces paramètres d'évolution, une analyse historique de l'évolution du trait de côte à travers plusieurs périodes de temps, sur la base de photos aériennes, d'ortho-photos et des relevés au DGPS, montre que les plages sont en perpétuel mouvement. À l'échelle historique (1948-2012), 15 plages sont considérées en recul, 16 sont stables, les autres montrent une avancée du trait de côte. Certaines périodes sont particulièrement importantes en termes de recul, comme celle de 1996 à 2002. L'évolution récente (20072012) permet de relativiser l'analyse historique en montrant les tendances actuelles de l'évolution. 23 plages sont en recul, 4 sont stables et 16 plages sont en avancée. Plusieurs paramètres doivent être pris en considération pour expliquer le comportement des plages tels que : l'orientation de la plage, la fermeture, la géologie, le régime hydroclimatique, la granulométrie et l'étendue. Les chroniques de houle modélisées dans le cadre d'ANEMOC ont été utilisées pour analyser les caractéristiques de la houle, la fréquence et la direction des événements de tempête les plus importants. Cette base de données complète a été utilisée pour obtenir un modèle conceptuel décrivant les facteurs les plus importants impliqués dans l'évolution côtière observée : rotation symétrique ou asymétrique de la plage, recul ou progradation homogène ainsi que les évolutions rapides en raison de la présence des banquettes de posidonie sur la plage.

Mots-clés : Plage de poche, Hydrodynamique, Houle, Sédiments, Dynamique côtière, Trait de côte, Littoral. 


\section{Thème 2 - Dynamique sédimentaire}

\section{Introduction}

La majorité du littoral mondial est caractérisé par des côtes rocheuses. Le long de ces côtes, la morphologie typique des plages de poche se caractérise par la présence de caps rocheux à leurs extrémités (SHORT \& MASSELINK, 1999). Ces plages sont généralement de faible étendue connues comme étant incurvées en fond de baie. Des études détaillées de ces plages de poche ont été menées (HSU \& EVANS, 1989 ; KLEIN et al., 2002 ; BOWMAN et al., 2009 ; DALY et al., 2011, VAN DE LAGEWEG et al., 2013). Il est largement admis que le comportement morphologique de ces plages est déterminé par leur orientation, leur indentation, leur pente, la granulométrie, la présence de barres sédimentaires sous-marines. L'évolution morphologique de ces systèmes est également dépendante du régime de la houle qui influence à la fois l'hydrodynamisme à la côte (SHORT \& MASSELINK, 1999) et les processus de rotation de la plage.

L'approche intégrant la géométrie de la plage (HSU et al., 1989) a été largement utilisée pour obtenir un premier aperçu du comportement des plages de poche. Cette approche est basée sur le principe que l'équilibre de la plage résulte en une courbe qui peut être définie par une fonction logarithmique, parabolique ou de puissance. Cette méthode permet ainsi d'évaluer l'état d'équilibre d'une plage de poche en évaluant la relation entre son indentation ( $\mathrm{a} / \mathrm{Ro}$ ) et l'incidence de la houle $\beta$.

Plusieurs études ont montré que les modèles utilisant des paramètres environnementaux généraux n’étaient pas assez précis pour expliquer clairement l'évolution morphologique des plages de poche (LOUREIRO et al., 2009, 2013). Les approches paramétriques semblent mieux expliquer le comportement des plages réflectives. La classification des plages utilisant la vitesse de chute des sédiments (WRIGHT \& SHORT, 1984) fournit également des informations supplémentaires sur l'applicabilité des approches géométriques, et donnent notamment une information sur la dérive littorale (SHORT \& MASSELINK, 1999).

L'objectif de cette contribution est d'évaluer l'impact de la géométrie sur l'évolution morphologique sur 44 plages de poche de la Corse.

\section{Domaine d'étude}

Le site d'étude est le département de la Haute-Corse situé dans la mer Méditerranée (figure 1). Le littoral est divisé en une côte sableuse à l'est et une longue côte rocheuse au nord où l'évolution morphologique de 44 plages de poche avec une exposition variable est observée. L'amplitude de la marée est d'environ 0,2 m et peut atteindre 0,3 $\mathrm{m}$ lors des grandes marées. Les conditions de houles sont faibles (Hs moyen $=0,8 \mathrm{~m}$; Ts moyen 4,5 s) mais les conditions de forte houle et les tempêtes d'hiver sont tout de même assez fréquents. La période de retour annuelle pour la houle est d'environ 4,7 m, mais des hauteurs significatives de houle de plus de $8 \mathrm{~m}$ ont été observées. La plupart des sites étudiés sont des plages réflectives composées de sables dont la granulométrie 


\section{XIII ${ }^{\text {èmes }}$ Journées Nationales Génie Côtier - Génie Civil \\ Dunkerque, 2-4 juillet 2014}

varie. Pour la plupart des sites, le taux d'érosion des falaises à proximité est faible et l'apport de sédiments est donc très limité. Les falaises le long du Cap Corse sont composées de complexes ophiolitiques, tandis que la côte ouest est formée de roches granitiques riches en magnésium et potassium.

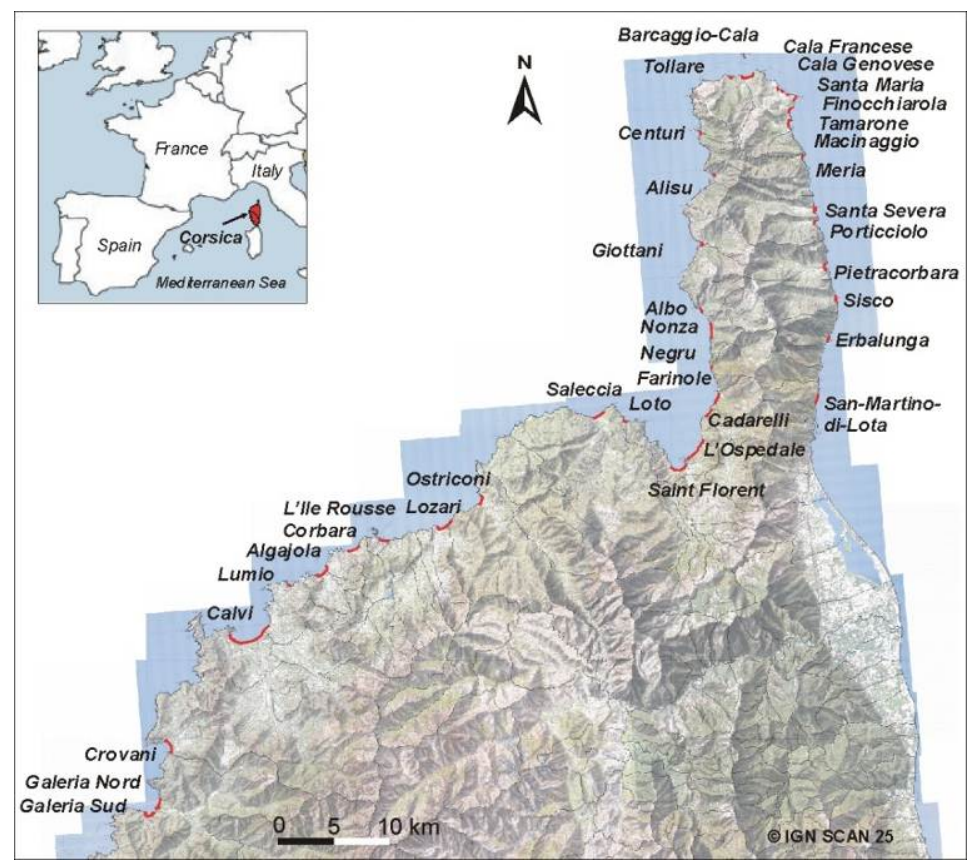

Figure 1. Localisation du domaine d'étude et des 44 plages de poche de Haute-Corse.

\section{Méthodologie}

\subsection{Les conditions hydrodynamiques}

Les mesures de la houle sont très occasionnelles le long du littoral de la Corse, et la plupart des données ne dispose pas d'information directionnelle. Afin d'analyser les caractéristiques du climat des houles et des tempêtes, la base de données ANEMOC (C) CETMEF / EDF R \& D LNHE-2010/2011) a été utilisée. ANEMOC (Atlas Numérique d'Etats de Mer Océanique et Côtier, http://anemoc.cetmef.developpementdurable.gouv.fr) a été construit à partir de simulations rétrospectives sur une période de 30 ans à partir de 01/01/1979 jusqu'au 31/12/2008 pour la façade méditerranéenne. Cinq bouées virtuelles (MEDIT 4655, 6530, 6771, 7846, 8110) ont été utilisées pour analyser le climat des houles le long du littoral de la Haute-Corse. Le climat moyen a été obtenu ainsi que les caractéristiques de tempêtes : la hauteur de houle maximale, l'énergie cumulée, la fréquence, la durée, le run-up et le set-up max à l'aide de la formule de STOCKDON et al. (2006). Un événement de tempête le long de cette côte est défini par une hauteur de houle significative supérieure à $2 \mathrm{~m}$ (ce qui est équivalent à plus de deux fois la hauteur moyenne des vagues). 


\section{Thème 2 - Dynamique sédimentaire}

\subsection{Morphologie des plages et évolution du trait de côte}

Une analyse de l'évolution du trait de côte a été réalisée à l'aide de photographies aériennes disponibles depuis 1948 (1948, 1951, 1960, 1983, 1985, 1996, 2002, 2007). Comme la limite supérieure de la plage est très souvent une falaise ou des infrastructures anthropiques, seule la limite sec/mouillé a été utilisée dans cette étude pour évaluer l'évolution à long terme du littoral. Compte tenu de la grande variabilité de cet indicateur et de la résolution des images, la position du trait de côte a une précision de $\pm 10 \mathrm{~m}$ pour les photographies anciennes (avant 1996) et $\pm 5 \mathrm{~m}$ pour les récentes. Certaines des plages étudiées sont suivies chaque année depuis 2000 dans le cadre du Réseau d'Observation du Littoral de la Corse (ROL, BALOUIN et al., 2013), ce qui a permis d'évaluer les tendances à court terme dans l'évolution de la position du trait de côte. Une mesure de la position du trait de côte au DGPS a été réalisée en 2012 sur l'ensemble des plages, ainsi que des profils topographiques, un échantillonnage des sédiments présents, des photographies ainsi que des observations de la géomorphologie (végétation, des herbiers litière, la présence d'une berme développé, pointes de plage, des bars côtiers).

Tous les traits de côte ont été numérisés dans le logiciel ARCGIS ®. Le module DSAS développé par l'USGS (THIELER et al., 2009) a été utilisé pour mesurer l'évolution du trait de côte dans le temps ainsi que les vitesses d'évolution associées (voir exemple sur la figure 2). Les caractéristiques des plages de poche ont été évaluées à la fois par des photographies aériennes et des analyses des données de terrain : l'orientation de la plage, la pente, la longueur, l'exposition, l'indentation, la fermeture, la géologie, la présence d'herbiers de posidonies. Ces informations permettent de calculer des indicateurs morphodynamiques (WRIGHT \& SHORT, 1984) et l'indicateur de stabilité (HSU \& EVANS, 1989). Une description du comportement morphologique a été définie pour les 44 plages qui distingue les plages stables, celles qui subissent une rotation unidirectionnelle, celles dont la rotation se fait dans les 2 sens, celles dont le recul ou l'avancée du trait de côte se fait de manière homogène.

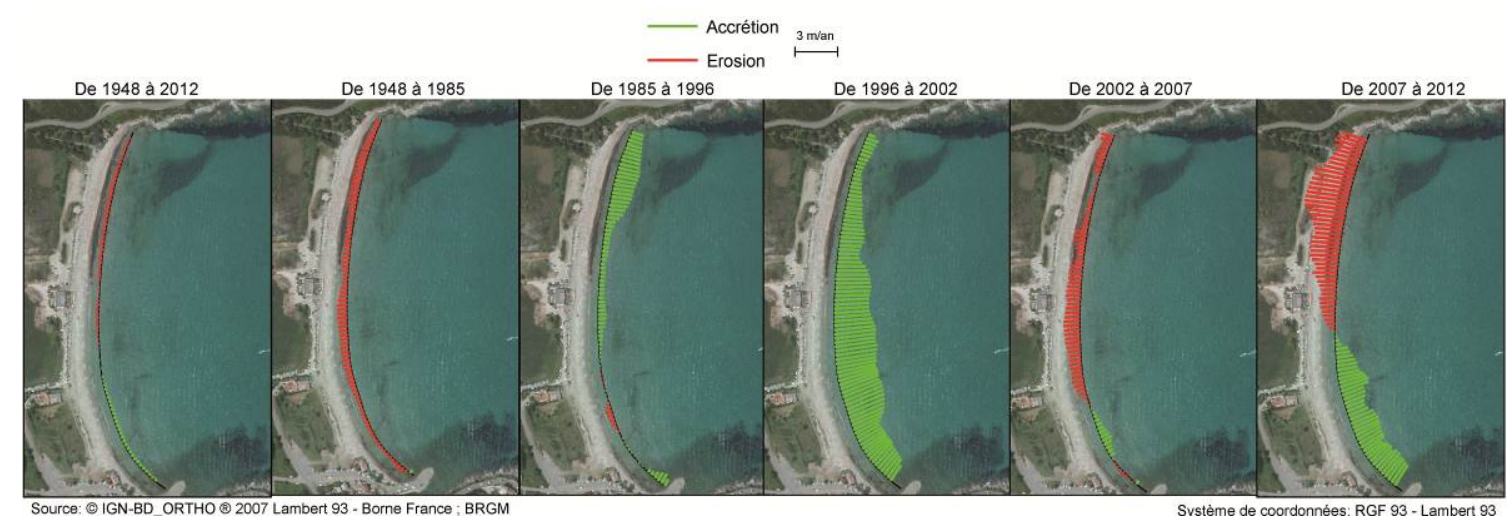

Figure 2. Exemple de l'évolution de la position du trait de côte entre 1948 et 2012 sur la plage de Pietracorbara sur la côte est du Cap Corse. 


\section{XIII ${ }^{\text {èmes }}$ Journées Nationales Génie Côtier - Génie Civil \\ Dunkerque, 2-4 juillet 2014}

\section{Résultats}

4.1 Les conditions hydrodynamiques

La base de données ANEMOC a été utilisée afin d'obtenir le climat de houle moyen ainsi que les caractéristiques des tempêtes au cours de la période 1979-2008 le long du littoral de la Haute-Corse. Les conditions particulières de tempêtes ont été extraites pour toutes les plages en fonction de leur orientation et de leur ouverture. La figure 2 illustre la direction de la houle dominante. Plus de 750 événements avec Hs $>2 \mathrm{~m}$ ont été analysés. La plupart des tempêtes ont des hauteurs significatives de houle autour de 3 m. mais certaines atteignent plus de 6 m comme celle observée en Novembre 2004. La durée moyenne des événements est d'environ 21 heures avec toutefois une durée maximale de 113 heures atteinte lors d'un épisode en Février 1990. Pour la plupart des plages, il y a une direction de tempête dominante. Cependant, de plus petits événements peuvent se produire avec une direction opposée contribuant ainsi à l'alternance dans la direction de transport des sédiments et les processus de rotation de la plage le long de la période étudiée. En outre, même si les caractéristiques de tempête sont comparables pour toutes les plages avec une énergie légèrement plus élevée sur la côte ouest où le fetch est plus long, la fréquence des tempêtes est plus importante sur le nord et la côte occidentale qu'à l'est. Alors que moins de 500 événements de tempêtes (Hs $>2 \mathrm{~m}$ ) ont été enregistrées sur la côte est, plus de 1000 ont été enregistrées sur la côte nord-est au cours de la période 1979-2008. Pour calculer le climat de houle moyen, la direction dominante a été utilisée (voir $\Omega$ et l’incidence sur la figure 3).

\subsection{L'évolution du trait de côte}

Sur le long terme (1948-2012), très peu de plages ont avancé. Les plages de Galeria Nord et Cala Genovese (voir localisation sur la figure 1) montrent une avancée respective de $20 \mathrm{~m}$ et $15 \mathrm{~m}$. Deux plages de l'ouest du Cap Corse, Albo et Nonza, présentent un élargissement très fort pour atteindre plus de 300 m entre 1948 et 1960. Cette évolution est cependant d'origine anthropique et découle des activités minières au voisinage de ces baies (Mine de Canari) et du dépôt de grandes quantités de matières extraites de la mine. 16 plages sont stables, 11 en recul et 4 en fort recul : Tollare, Giottani et Negru où le recul atteint $40 \mathrm{~m}$ et Saleccia où un recul de $60 \mathrm{~m}$ a été observé dans la partie sud de la baie.

L'évolution récente, de 2007 à 2012, donne la tendance de l'évolution actuelle, sur toutes les plages étudiées, 4 sont stables, 16 sont en avancée et 23 sont en recul. Cette évolution est beaucoup plus contrastée que l'évolution historique pour laquelle, 13 plages sont en avancée importante et 16 sont marquées par un recul.

L'analyse de l'évolution du trait de côte historique montre des comportements contrastés des plages exposées aux mêmes forçages, ce qui implique une importance des paramètres locaux. L'évolution à court terme (voir la figure 3) montre la variabilité temporelle du processus d'érosion. Ceci est particulièrement le cas pour les plages où 


\section{Thème 2 - Dynamique sédimentaire}

une banquette de posidonies recouvre la plage (figure 3). Le volume de cette banquette est très variable dans le temps en raison de l'arrachage de l'herbier lié aux conditions de houle, ainsi qu'au fait que certaines de ces plages sont "nettoyées" avant la saison estivale.

Certaines périodes montrent un recul du trait de côte très rapide, comme pendant 19962002 le long de la côte orientale du Cap Corse. Les conditions de houle au cours de cette période ne sont pas plus énergiques, mais la durée entre deux événements successifs a été légèrement réduite, empêchant la récupération de la plage, ce qui pourrait expliquer le fort recul observé.

\subsection{La morphodynamique}

Pour comprendre les différences dans l'évolution du littoral des plages de poche corses, plusieurs paramètres ont été analysés (voir figure 3). Le premier paramètre est l'incidence de la houle à $10 \mathrm{~m}$ de profondeur.

Compte tenu des directions dominantes de la houle, l'incidence de la houle est très variable. Du fait de leur orientation, les plages de la côte est sont beaucoup plus exposées aux houles très obliques. Dans de tels cas, la réfraction des ondes est plus importante, et l'énergie est supposée être inférieure à la côte.

Le nombre de tempêtes au cours des 30 dernières années est également plus faible que sur la côte nord. Ce climat de houle est en accord avec le comportement morphologique principal de ces plages qui se caractérise par la rotation de la plage qui peut être soit unidirectionnelle ou bidirectionnelle compte tenu de l'orientation et de la fermeture de la plage. Sur la côte ouest du Cap, les événements de tempêtes les plus importants atteignent la côte avec une très faible incidence. Le comportement morphologique principal observé est un recul homogène du trait de côte, à l'exception des plages dont l'évolution dépend de facteurs anthropiques, Nonza et Albo. Sur le reste de la côte, la direction de la houle est principalement normale à la côte, mais l'orientation des plages est beaucoup plus variable et il est difficile de définir une tendance générale.

Afin de tenir compte de la morphologie des baies, l'état de la stabilité des plages utilisant le rapport d'indentation a/Ro et les angles $\beta$ des crêtes de vague ont été examinés (voir les figures 3 et 4). Ce concept est largement utilisé pour évaluer dans une première phase l'état d'équilibre des plages. Les résultats (figure 4) indiquent que la plupart des plages de la côte orientale sont instables. La principale raison en est la forte incidence de la houle, en particulier lors des tempêtes du sud-est. Ces plages, et quelques autres le long du littoral, sont généralement de faible étendue, fortement indentée et sont connues pour être recouverte par une grande quantité de feuilles de Posidonia oceanica. Malgré cet état apparent d'instabilité, elles ont montré une position assez stable de leur trait de côte au cours des dernières décennies. 


\section{XIII ${ }^{\text {èmes }}$ Journées Nationales Génie Côtier - Génie Civil \\ Dunkerque, 2-4 juillet 2014}
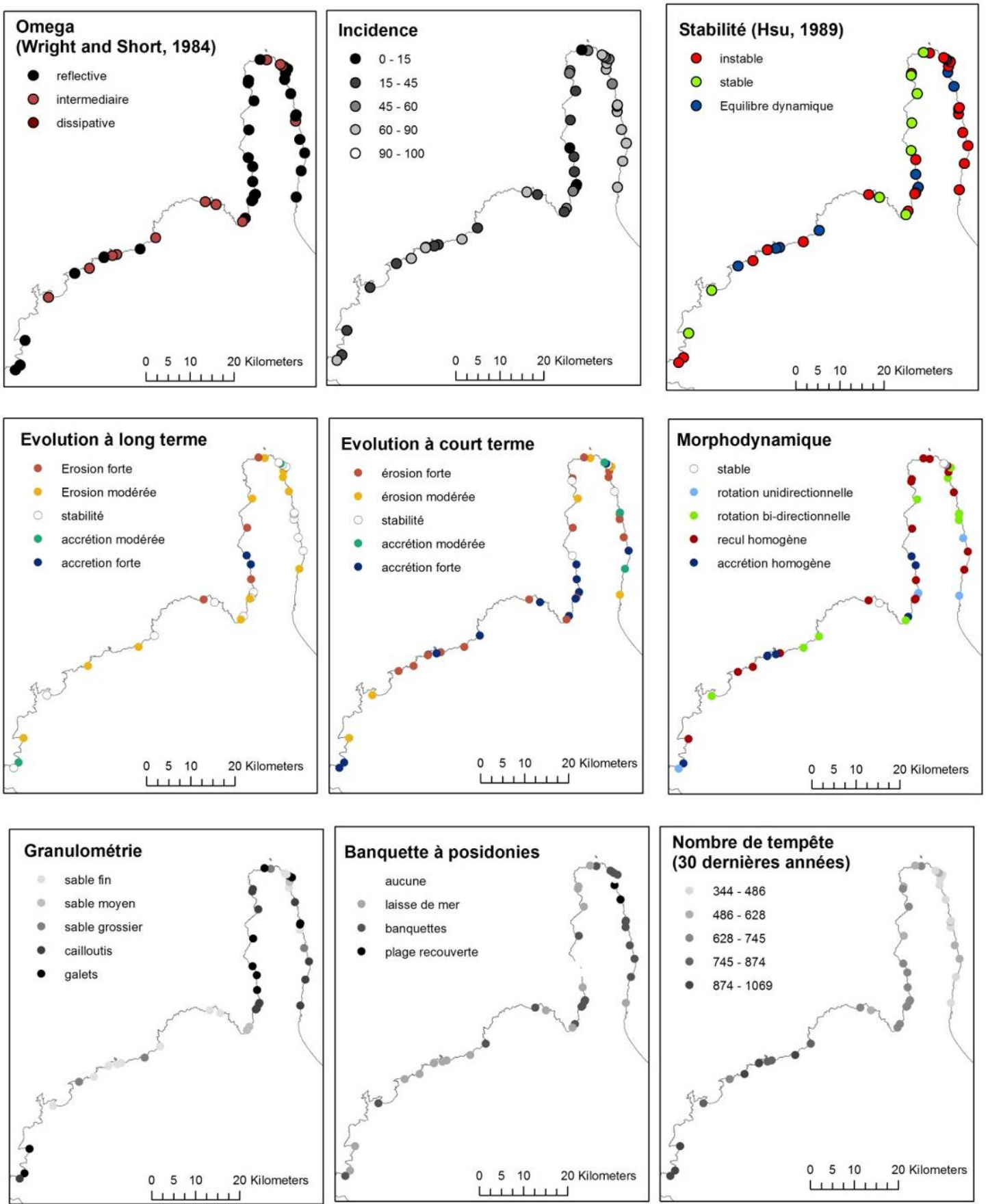

Figure 3. Indicateurs morphologiques obtenus sur les 44 plages de Haute-Corse. En haut : la vitesse de chute $\Omega$; l'incidence de la houle à $10 \mathrm{~m}$ de profondeur ; les résultats du test de stabilité (HSU \& EVANS, 1989). Au milieu : l'évolution à long terme du trait de côte (1948-2012) ; l'évolution à court terme (2007-20012); les principaux processus morphologiques observés. En bas : la granulométrie; la présence de banquette de posidonies ; le nombre d'évènements dont Hs>2 m entre 1979 et 2009. 


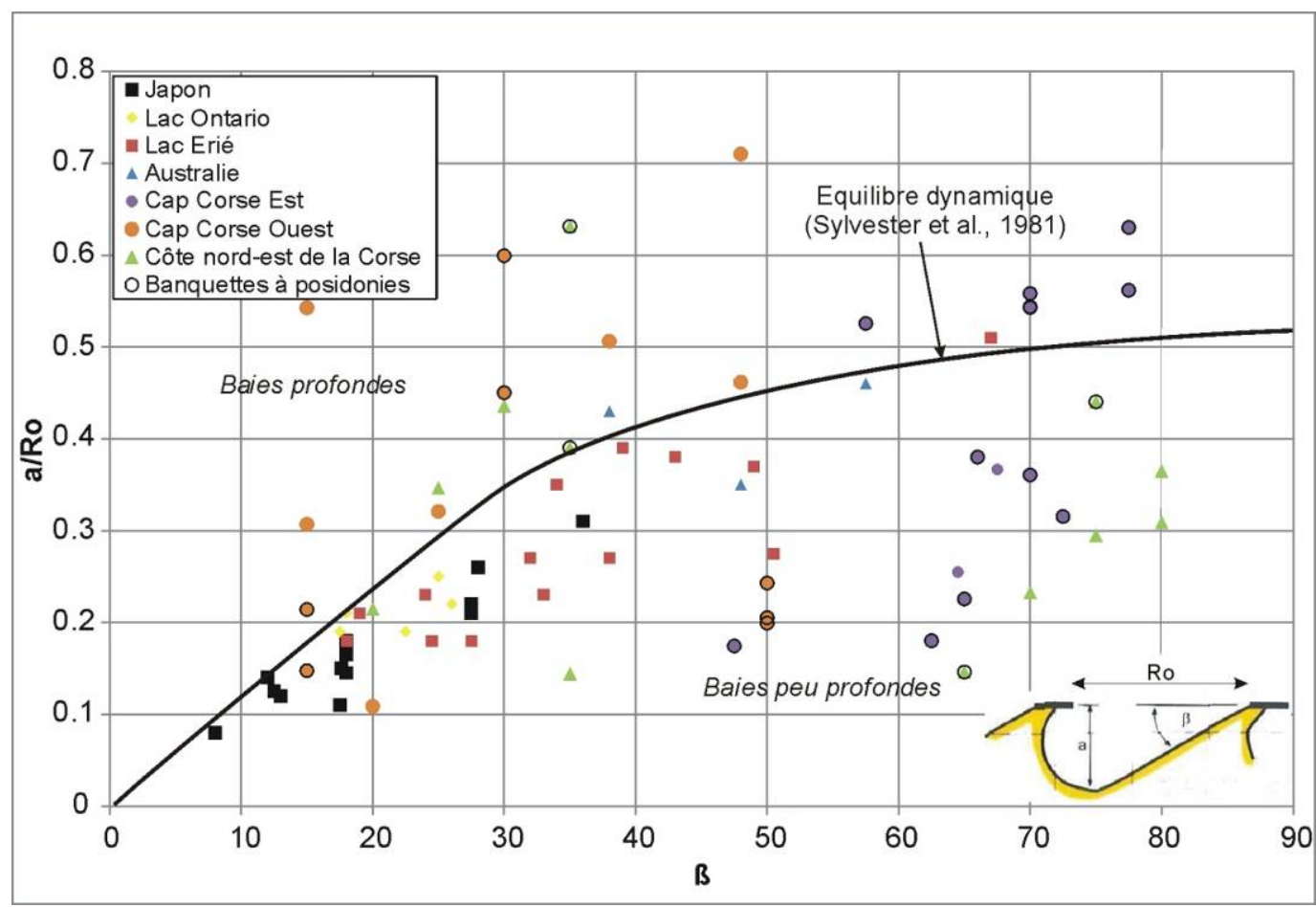

Figure 4. Relation entre le rapport d'indentation ( $(\mathrm{a} / \mathrm{Ro})$ et l'angle entre la houle incidente et la ligne joignant le cap rocheux à la plage à partir duquel la houle subit le phénomène de diffraction (modifié à partir de BISHOP, 1983 dans SIMEONI et al., 2012). Le contour noir des points indique la présence importante de feuilles de posidonies sur la plage.

\section{Discussion}

Les résultats sur l'évolution de la position du trait de côte et les changements morphologiques des 44 plages de poche de Haute-Corse révèlent des comportements contrastés, même pour les plages soumises aux mêmes conditions de forçage. L'orientation de la plage affecte de manière significative l'évolution du trait de côte, avec un recul marqué lorsque l'incidence de la houle est faible. Ceci est particulièrement le cas pour les plages de Tollare, Giottani, Saint- Florent, Lumio. Cependant, plusieurs plages, comme l'Ostriconi, qui bien qu'elles aient une faible incidence et sont exposées à des tempêtes de fortes intensités, restent assez stables sur le long terme.

Comme il a été observé par DAI et al. (2010), et numériquement par DALY et al. (2011), les plages stables sont sujettes à une plus grande oscillation de la plage (mouvement vers la côte ou vers le large) que celles en rotation. Ceci a été observé sur plusieurs plages (Meria, l'Ile Rousse, Corbara, Lumio). L'étalement directionnel de la houle est très large pour toutes les plages subissant une rotation bidirectionnelle (voir sur la figure 2). Cependant, seulement 13 plages ont une tendance marquée de rotation sur le littoral observé. En dépit de l'apparition de tempêtes de direction de provenance opposée, certaines de ces plages présentent une alternance du sens de rotation, tandis 


\section{XIII ${ }^{\text {èmes }}$ Journées Nationales Génie Côtier - Génie Civil \\ Dunkerque, 2-4 juillet 2014}

que d'autres présentent une évolution unidirectionnelle, avec une partie de la baie fournissant toujours des sédiments à l'autre. Bien sûr, cette observation se fait sur plusieurs sites et l'évolution à court terme en relation avec les événements de tempête n'a pas été prise en compte et peut montrer un comportement très différent entre l'évolution due à la tempête ainsi que de la tendance à moyen et à long terme.

L'analyse de stabilité a montré que seulement 9 des 44 plages étudiées ont une forme stable. La plupart de ces plages sont sur la côte ouest du Cap Corse et se caractérisent par une faible incidence de la houle. Les plages qui sont censées être stables (en vert sur la figure 3) présentent au cours de la dernière décennie une tendance au recul, mais selon l'ensemble des données disponibles, ne montrent pas de modification importante de leur forme. Sur ces sites, le recul concerne toute la baie et aucun processus de rotation n'est visible sur le littoral concerné. Les plages en équilibre dynamique selon la classification de HSU et al. (2008), 8 plages sur 44, ont comme prévu une tendance au recul sur le long terme qui peut être soit un recul homogène ou un processus de rotation de la plage qui entraine une diminution de la surface de la plage.

Pour ces deux catégories, stables et en équilibre dynamique, l'analyse de la stabilité a tendance à donner de bons résultats et à expliquer globalement l'évolution du trait de côte, même si le taux de recul du rivage est très variable et dépend de paramètres morphologiques locaux (orientation de la plage, longueur de la plage).

Les plages instables représentent la majorité des plages de poche de Haute-Corse (27 sur 44). Leur dynamique et leur évolution est très variable. Sur la côte est, où les tempêtes sont moins fréquentes et l'incidence de la houle plus élevée, l'évolution à long terme du trait de côte est soit stable ou en recul modéré, mais l'évolution à court terme indique une grande variabilité temporelle. Même si les événements de tempêtes sont moins fréquents et plus réfractés, ces plages ont des évolutions morphologiques très importantes durant les tempêtes, comme en 2003, lorsque la route longeant la plage de Porticciolo a été partiellement détruite par les vagues déferlantes. L’évolution de ces plages est difficilement prévisible par les classifications actuellement utilisées. Cependant, les caractéristiques de ces plages et leur exposition est tout à fait comparable à celle des autres et leur seule caractéristique commune est la présence d'herbiers de posidonies en bas de plage, et pour certaine sur la totalité de la plage.

Cette couverture biologique a un rôle très important sur l'évolution du trait de côte : quand une quantité importante de feuilles est présente sur la plage et dans la zone du jet de rive, elle protège la plage des houles de tempête. Cependant, une tempête peut avoir un double impact sur la quantité de feuilles présentes : les houles peuvent à la fois favoriser le dépôt de feuilles sur les plages, mais également les enlever lors d'évènements plus énergétiques augmentant ainsi la vulnérabilité de la plage face aux tempêtes suivantes. Pour ces plages, la classification de la stabilité ne reflète bien évidemment pas l'état réel de la plage. Les feuilles de posidonies ont un comportement très particulier. Quand seulement quelques feuilles sont sur le rivage, elles ont tendance 


\section{Thème 2 - Dynamique sédimentaire}

à être facilement déplacées par les vagues, mais quand elles forment une banquette, leur structure est plus solide et seuls les événements les plus énergétiques peuvent les déplacer. En outre, lorsque les feuilles sont dispersées dans l'eau près de la côte, elles donnent à l'eau un comportement plus visqueux qui atténue l'énergie des vagues à la côte. Dans cette étude, l'ensemble des données disponibles n'était pas suffisant pour bien comprendre le rôle de l'herbier et de sa dynamique (quand elle est déposée / érodée, et pourquoi ?). Toutefois, la surveillance en temps réel en utilisant des systèmes de vidéo, pourrait être utile à déterminer la dynamique de ces plages qui représentent la majorité des plages corses.

\section{Conclusion}

Une base de données sur 44 plages de poche de Haute-Corse a été obtenue, décrivant l'évolution du trait de côte à moyen et long terme, les caractéristiques locales des plages et l'exposition aux houles. L'analyse de stabilité de HSU et al. a été appliquée pour évaluer la pertinence d'une telle méthode en Corse. L'étude indique que cette méthode donne des résultats tout à fait intéressants pour prédire le comportement des plages stables et celles en équilibre dynamique. Cependant, pour la plupart des plages, la méthode n'est pas applicable en raison de la présence d'herbiers de posidonies qui modifie totalement la morphodynamique. D'autres études sur l'impact de ces herbiers sur la position du trait de côte sont nécessaires pour être en mesure d'anticiper l'évolution des plages de poche corses.

\section{Remerciements}

Les travaux décrits dans la présente publication ont été soutenus par la Direction Départementale du Territoire et de la Mer de la Haute-Corse. Pour cette étude, les résultats de la modélisation issus de la base de données ANEMOC (EDF R \& D et le CETMEF) ont été utilisés.

\section{Références bibliographiques}

BALOUIN Y., STEPANIAN A., BELON R. (2013). The Corsican coastal monitoring network. In: Coastal erosion monitoring - a network of regional observatories. Ed. L.E. Cipriani. Nuova grafica fiorentina.

BISHOP C.T. (1983). A shore protection alternative: artificial headlands. Proc. Candian Coastal Conf., NRCC, pp 305-319.

BOWMAN D., GUILLEN J., LOPEZ L., PELLEGRINO V. (2009). Planview geometry and morphological characteristics of pocket beaches on the catalan coast (Spain). Geomorphology, Vol. 108, pp 191-199. http://dx.doi.org/10.1016/j.geomorph.2009.01.005 


\section{XIII ${ }^{\text {èmes }}$ Journées Nationales Génie Côtier - Génie Civil \\ Dunkerque, 2-4 juillet 2014}

DALY C.J., BRYAN K.R., ROELVINK J.A., KLEIN A.H.F., HEBBELN D., WINTER C. (2011). Morphodynamics of embayed beaches: the effect of wave conditions. J. of Coast. Res., Vol. SI64, pp 1003-1007.

DAI Z., LIU J.T., LEI Y.P., ZHANG X.L. (2010). Patterns of sediment transport pathways on a headland beach - Nawan Beach, South China: a case study. J. of Coast. Res., Vol. 26(6), pp 1096-1103. http://dx.doi.org/10.2112/JCOASTRES-D-09-00097.1

HSU J.R.C., EVANS C. (1989). Parabolic bay shapes and applications. Inst. Civ. Eng. Proc., London, Vol. 87, pp 556-570.

HSU J.R.C., SILVESTER R., XIA Y.M. (1989). Generalities on static equilibrium bays. Coastal Engineering, Vol. 12, pp 353-369. http://dx.doi.org/10.1016/0378-3839(89)90012-4

KLEIN A.H.F., FILHO L.B., SCHUMACHER D.H. (2002). Short-term beach rotation processes in distinct headland bay beach systems. J. Coast. Res., Vol. 18, pp 442-458.

LOUREIRO C., FERREIRA O., COOPER J.A.G. (2009). Contrasting Morphologic behavior at embayed beaches in Southern Portugal. J. of Coast. Res., Vol. SI56 pp 83-87.

LOUREIRO C., FERREIRA O., COOPER J.A.G. (2013). Applicability of parametric beach morphodynamic state classification on embayed beaches. Marine Geology, Vol. 346, pp 153-164. http://dx.doi.org/10.1016/j.margeo.2013.09.005

SHORT A.D., MASSELINK G. (1999). Embayed and structurally controlled beaches. In: Short A.D. (Ed.), Handbook of beach and shoreface dynamics. John Wiley and Sons, Chichester, pp 230-249.

SIMEONI U., CORBAU C., PRANZINI E., GINESU S. (2012). Le Pocket Beach. Dinamica e gestione delle piccole spiagge. Ed FrancAngeli, 171 p.

STOCKDON H.F, HOLMAN R., HOWD P., SALLENGER JR. A.H. (2006). Empirical parametrization of setup, swash and runup. Coastal Engineering, Vol. 53, pp 573-588. http://dx.doi.org/10.1016/j.coastaleng.2005.12.005

THIELER E.R., HIMMELSTOSS E.A., ZICHICHI, J.L., ERGUL A. (2008). Digital Shoreline Analysis System (DSAS) version 4.0.An ArcGIS extension for calculating shoreline change: U.S. Geological Survey Open-File Report 2008-1278.

VAN DE LAGEWEG W.I., BRYAN K.R., COCO G., RUESSINK B.G. (2013). Observation of shoreline-sandbar coupling on an embayed beach. Marine Geology, Vol. 344, pp 101-114. http://dx.doi.org/10.1016/j.margeo.2013.07.018 
Thème 2 - Dynamique sédimentaire 